\title{
Effects of 42 months of GH treatment on bone mineral density and bone turnover in GH-deficient adults
}

\author{
Matti J Välimäki, Pasi I Salmela ${ }^{1}$, Jorma Salmi ${ }^{2}$, Jorma Viikari ${ }^{3}$, Matti Kataja ${ }^{4}$, Heidi Turunen ${ }^{5}$ and Esa Soppi ${ }^{5}$ \\ Department of Medicine, University of Helsinki, Helsinki, Finland, ${ }^{1}$ Department of Medicine, University of Oulu, Oulu, Finland, \\ ${ }^{2}$ Department of Medicine, University of Tampere, Tampere, Finland, ${ }^{3}$ Department of Medicine, University of Turku, Turku, Finland, \\ ${ }^{4}$ National Public Health Institute, Helsinki, Finland and ${ }^{5}$ Pharmacia E Upjohn Oy, Medical Department, Vantaa, Finland \\ (Correspondence should be addressed to M Välimäki, Department of Medicine, Helsinki University Central Hospital, FIN-O0290 Helsinki, Finland)
}

\begin{abstract}
Objective: To study the effects of GH treatment for up to 42 months on bone mineral density (BMD) and bone turnover.

Design and methods: BMD with dual energy X-ray absorptiometry, serum type I procollagen carboxyterminal propeptide (PICP), serum type I collagen carboxy-terminal telopeptide (ICTP) and serum IGF-I were assessed in 71 adults with GH deficiency. There were 44 men and 27 women, aged 20 to 59 (median 43) years. Thirty-two patients completed 36 months and 20 patients 42 months of treatment.

Results: The BMD increased for up to 30-36 months and plateaued thereafter. In the whole study group, the maximum increase of BMD was $5.0 \%$ in the lumbar spine $(P<0.001), 5.9 \%(P<0.01)$ in the femoral neck, $4.9 \%$ (NS, $P>0.05)$ in the Ward's triangle and $8.2 \%(P<0.001)$ in the trochanter area. The serum concentrations of PICP $(202.6 \pm 11.5$ vs $116.3 \pm 5.4 \mu \mathrm{g} / \mathrm{l}$; mean \pm S.E.M. $)$ and ICTP $(10.5 \pm 0.6$ vs $4.4 \pm 0.3 \mu \mathrm{g} / \mathrm{l})$ doubled $(P<0.001)$ during the first 6 months of $\mathrm{GH}$ treatment but returned to baseline by the end of the study $(130.0 \pm 10.4$ and $5.6 \pm 0.7 \mu \mathrm{g} / \mathrm{l}$ respectively), despite constantly elevated serum IGF-I levels $(39.6 \pm 4.1 \mathrm{nmol} / \mathrm{l}$ at $42 \mathrm{months} v \mathrm{~s}$ $11.9 \pm 0.9 \mathrm{nmol} / \mathrm{l}$ at baseline; $P<0.001)$. The responses to $\mathrm{GH}$ treatment of serum IGF-I, PICP, ICTP $(P<0.001$ for all; ANOVA $)$ and of the BMD in the lumbar spine $(P<0.05)$, in the femoral neck and the trochanter $(P<0.001$ for both) were more marked in men than in women. At the end of the study the BMD had increased at the four measurement sites by $5.7-10.6 \%(P<0.01-0.001)$ in patients with at least osteopenia at baseline and by $0.1-5.3 \%$ (NS $P<0.05)$ in those with normal bone status $(P<0.001$ for differences between groups; ANOVA). Among patients who completed 36-42 months of treatment, the number of those with at least osteopenia was reduced to more than a half. The response of BMD to GH treatment was more marked in young than in old patients at three measurement sites $(P<0.05-<0.001$; ANOVA). In the multiple regression analysis the gender and the pretreatment bone mass appeared to be independent predictors of three measurement sites, whereas the age independently determined only the vertebral BMD.

Conclusions: GH treatment in GH-deficient adults increased BMD for up to 30-36 months, with a plateau thereafter. Concurrently with the plateau in BMD the bone turnover rate normalized. From the skeletal point of view GH-deficient patients exhibiting osteopenia or osteoporosis should be considered as candidates for GH supplementation of at least 3-4 years.
\end{abstract}

European Journal of Endocrinology 140 545-554

\section{Introduction}

Bone mineral density (BMD) is reduced in adults with growth hormone (GH) deficiency (1), especially in those with childhood-onset disease (2), but also in subjects who developed the deficiency in adulthood (3-5). As a consequence of low BMD, GH-deficient adults have a doubled fracture rate compared with healthy controls (6).

$\mathrm{GH}$ replacement therapy in GH-deficient adults accelerates whole body bone remodeling, demonstrated not only by biochemical markers of bone resorption and bone formation (7-19) but also by histomorphometry (20). After an initial decline $(12-14,21,22)$ BMD has increased in studies in which treatment with GH has continued for 12 months or more $(14-16,18,19,21,23,24)$. The response of BMD to $\mathrm{GH}$ replacement therapy has been more marked in patients with childhood-onset rather than adultonset GH deficiency $(14,22,23)$, in male rather than female patients (18), and in those subjects with a low pretreatment bone mass $(18,24)$. 
In studies published to date the effect of $\mathrm{GH}$ treatment on BMD of the lumbar spine and the forearm has been followed for 4 years (25) and on that of the upper femur at longest only for 2 years (18). Thus, more long-term data are needed to show whether the increase in BMD continues or plateaus over time. In the present trial we followed the effects of $\mathrm{GH}$ replacement therapy on bone mass and markers of bone turnover for up to 42 months in a cohort of 71 adults with GH deficiency, the great majority of them having an adult-onset form of the disease.

\section{Subjects and methods}

\section{Patients}

Seventy-one adults (44 men, 27 women) with known pituitary deficiency of adult onset $(n=59)$ or childhood onset (age at diagnosis 16 years or less, $n=12$ ) and a median age of 43 years (range 20-59 years) participated in the trial conducted at four Finnish University Hospitals (Table 1). The median age at diagnosis of GH deficiency was 41 years (range 6-59 years). At the start of treatment 11 of 12 patients with childhoodonset GH deficiency were aged 30 years or less. The diagnosis of GH deficiency was based upon a maximum peak GH response (assayed by polyclonal competitive RIAs) of less than $10 \mathrm{mU} / \mathrm{l}(5 \mu \mathrm{g} / \mathrm{l})$ to stimulation by glucagon $(70 \%$ of the patients) or clonidine $(27 \%)$, or insulin-induced hypoglycemia (3\%). Patients with childhood-onset disease were retested at the beginning of the study. The median known duration of $\mathrm{GH}$ deficiency before the trial was 1.8 (range 0-39) years. When needed, patients received adequate and stable replacement therapy with glucocorticoids, thyroxine, gonadal steroids and desmopressin. Ninety-four percent of the patients used at least one of these replacement therapies (Table 1), which were kept constant during the entire study period. Before the trial they had used replacement therapies for 1-27 (median 8) years.

\section{Study protocol}

Patients were entered in a 6 month randomized doubleblind trial of either GH or placebo. Subsequently, they were enrolled into an open trial for 12 months, after which the patients were offered an opportunity to continue GH treatment; 46 decided to do so. During the first 4 weeks of treatment the daily GH (Genotropin, Pharmacia \& Upjohn Oy, Vantaa, Finland) dose was $0.018 \mathrm{IU}(6 \mu \mathrm{g}) / \mathrm{kg}$ body weight (BW) as an s.c. injection at bedtime, and the target dose thereafter was $0.036 \mathrm{IU}$ $(12 \mu \mathrm{g}) / \mathrm{kg}$ BW daily $(2.5 \mathrm{IU} /$ day for a subject of $70 \mathrm{~kg}$ BW). The dose was reduced in the event of side-effects. At the start of the study and every subsequent 6 months, physical and laboratory examinations were performed, including measurements of BMD. The body mass index (BMI) was calculated as BW in kilograms divided by height in meters squared. For those patients who were randomized to placebo in the double-blind

Table 1 Clinical characteristics of 71 adult patients with GH deficiency.

\begin{tabular}{lccc}
\hline & All & Men & Women \\
\hline Number & 71 & 44 & 27 \\
Age (years) at start (median with range) & 43 & 42 & 44 \\
& $(20-59)$ & $(20-59)$ & $(22-58)$ \\
Number of patients with childhood-onset disease & 12 & 9 & 3 \\
36 months' treatment & 6 & 4 & 2 \\
42 months' treatment & 3 & 2 & 1 \\
Age (years) at diagnosis (median with range) & 41 & 39 & 43 \\
& $(6-59)$ & $(6-59)$ & $(11-58)$ \\
Known duration (years) of GH deficiency (median with range) & 1.8 & 3.3 & 1.1 \\
& $(0-39)$ & $(0-39)$ & $(0-16)$ \\
Cause of pituitary deficiency & & & 12 \\
Pituitary adenoma & 33 & 21 & 5 \\
Craniopharyngioma & 18 & 13 & 4 \\
Idiopathic & 8 & 4 & 6 \\
Other & 12 & 6 & 22 \\
Hormonal replacement therapy & & & 23 \\
Corticosteroids & 59 & 37 & 9 \\
Levothyroxine & 61 & 38 & 21 \\
Sex steroids & 59 & 41 & 1 \\
Desmopressin & 23 & 14 & 28 \\
Some other drug & 49 & 2 & \\
Isolated GH deficiency & 3 & &
\end{tabular}


phase of the study, data collected at the end of the placebo phase were taken as the basal values.

Informed written consent was obtained from all patients. The study was approved by the local ethical committees of Oulu, Tampere and Turku University Hospitals and that of Third Department of Medicine, Helsinki University Central Hospital.

\section{BMD measurement}

BMD at the lumbar spine and at three femoral sites (femoral neck, trochanter area, Ward's triangle) was measured by dual energy X-ray absorptiometry. A Hologic QDR-1000 (Hologic Inc., Waltham, MA, USA) densitometer was used in Helsinki and Turku, and Lunar DPX (Lunar Radiation Inc., Madison, WI, USA) equipment in Oulu and Tampere. The coefficients of variation $(\mathrm{CV})$ for precision of the BMD measurements for the densitometers used varied from 0.9 to $1.7 \%$ in the lumbar spine and from 1.1 to $2.5 \%$ in the femoral neck. To present BMD results, percent changes from baseline were calculated. Standardized T score analyses, which compare individual bone density determinations with those of a young normal population of the same gender, were used for definition of osteoporosis and osteopenia. According to the criteria defined by the WHO, T scores more than 2.5 s.D. below the mean of the young normal population represent osteoporosis, whereas $\mathrm{T}$ scores between -1.0 and -2.5 represent osteopenia (26).

\section{Biochemical assays}

Blood samples were drawn in the morning after an overnight fast. The serum concentration of insulin-like growth factor-I (IGF-I) was determined by RIA after acid-alcohol extraction (Nichols Institute, San Juan Capistrano, CA, USA) with intra- and interassay CV ranging from 3 to $9 \%$.

The serum concentrations of carboxy-terminal propeptide of type I procollagen (PICP) and the carboxyterminal cross-linked telopeptide of type I collagen (ICTP) were determined by RIA kits from Orion Diagnostica, Oulunsalo, Finland. The intra-assay and interassay $\mathrm{CV}$ for these assays ranged from 3 to $9 \%$.

\section{Statistics}

A paired $t$-test was used in testing the changes from baseline with the null hypothesis stating no change was to be expected. If the null hypothesis turned out to be wrong, the size of differences and relations between variables were modeled using stepwise multiple regression analysis. Differences in response to GH between women vs men, young vs old patients, and patients with osteopenia vs normal bone status were tested by ANOVA. Furthermore, stepwise linear regression analysis was used to determine independent predictors of the response in BMD to treatment. Those given for the program included gender, duration of treatment, pretreatment bone mass, age, age squared and 100/age. The contribution of each variable to the explained variance was calculated using standardized correlation coefficients ( $\beta$ coefficients).

\section{Results}

Fifty-eight patients completed the 18-month trial (1218 months on GH). During the first 18 months six patients were withdrawn from the study due to adverse effects (myalgia in three, edema in two, elevated blood glucose in one), one patient appeared to have mild diabetes even at entry (a protocol violation), four patients did not want to continue the study, and one patient was otherwise lost from the follow-up. One patient presented with meningioma. His pituitary tumor had been treated with surgery and radiation treatment 20 years before the trial; no computed tomography or magnetic resonance imaging study was performed at entry. From 18 months onwards all drop-outs were consent withdrawals. Thirty-two patients completed 36 months and 20 patients 42 months of treatment; a difference in these numbers is due to the placebo phase at the beginning of the study. The mean BMI remained unchanged throughout the study $\left(28.3 \pm 0.6 \mathrm{~kg} / \mathrm{m}^{2}\right.$ at entry, $29.8 \pm 1.1 \mathrm{~kg} / \mathrm{m}^{2}$ at 36 months, $29.6 \pm 1.2 \mathrm{~kg} / \mathrm{m}^{2}$ at 42 months; mean \pm s.E.M., NS).

\section{BMD}

At baseline the BMD was lower in patients with childhood-onset disease than in those with adult-onset GH deficiency in the trochanter $(P<0.05)$ but not in other measurement sites. During the first 6 months of $\mathrm{GH}$ treatment the BMD seemed to decline at all the four measurement sites, significantly so only at the femoral neck $(-0.9 \%, P<0.05)$ (Fig. $1 \mathrm{~A}-\mathrm{D})$. Significant increases in BMD were noticed at the trochanter area after 12 months, and at the lumbar spine, the femoral neck and the Ward's triangle after 18 months of GH treatment. The BMD in the lumbar spine increased by $3.4 \%(P<0.001)$ after 24 months, by $5.0 \%(P<0.001)$ after 36 months and by $4.8 \%(P<0.001)$ after 42 months of GH treatment (Fig. 1A). At the femoral neck the respective increases were $3.4(P<0.001), 5.9$ $(P<0.01)$ and $5.7 \%(P<0.01)$ (Fig. 1B). At the Ward's triangle the BMD increased by $4.0 \%(P<0.05)$ after 24 months, by $4.6 \%$ (NS) after 36 months and by $4.9 \%$ (NS) after 42 months of GH treatment (Fig. 1C). At the trochanter area the respective increases were $5.4(P<0.001), 7.8(P<0.001)$ and $8.2 \%(P<0.001)$ (Fig. 1D). As analyzed separately, the increases in BMD for the 20 patients who completed the 42 months of treatment were similar to those presented above for the whole study population (Fig. 1A-D). 

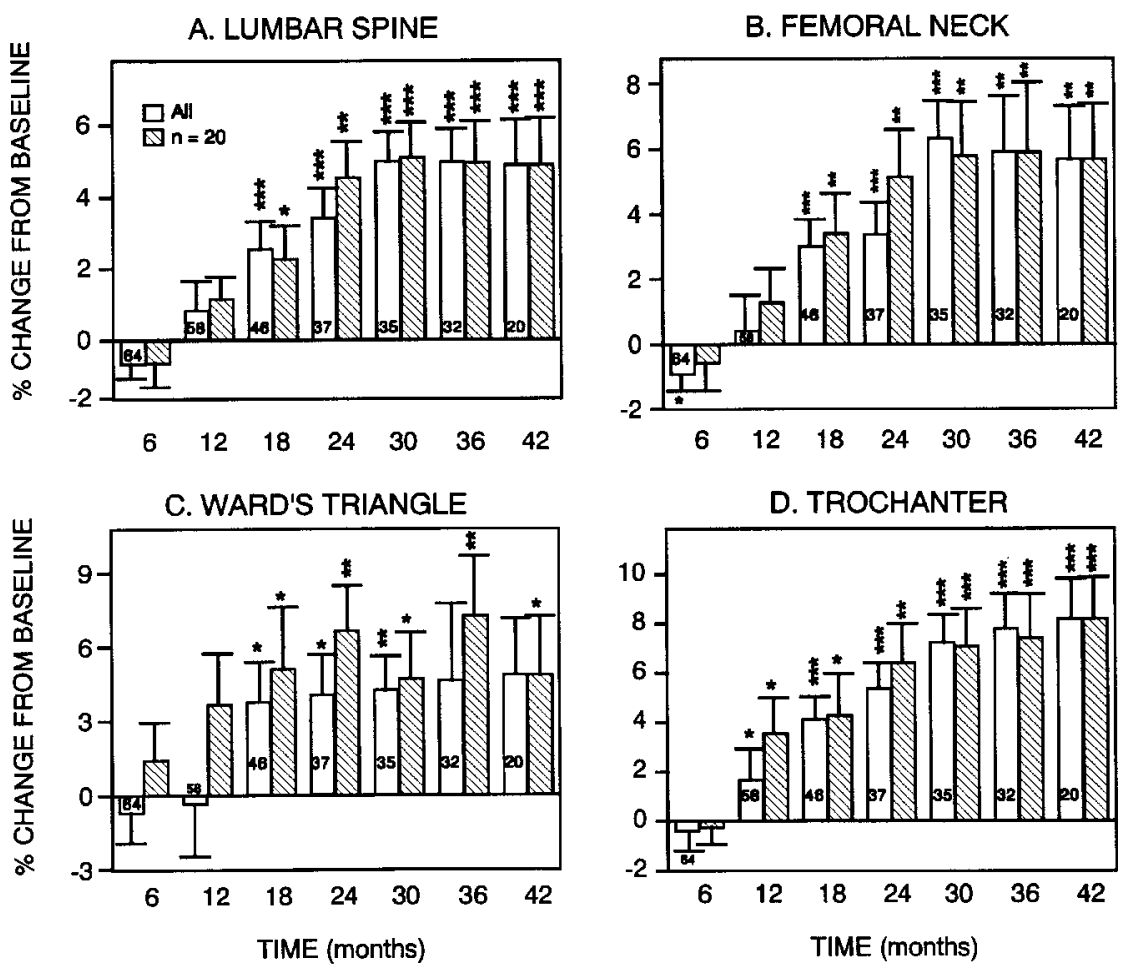

Figure $1 \mathrm{BMD}$ (mean \pm S.E.M.) in response to $\mathrm{GH}$ treatment of up to 42 months at the lumbar spine $(\mathrm{A})$, the femoral neck (B), the Ward's triangle $(C)$, and the trochanter area (D) in the whole study population and in the 20 patients who completed 42 months of treatment. ${ }^{\star} P<0.05,{ }^{* *} P<0.01,{ }^{* * *} P<0.001$ for differences from baseline. $n$ values in the white bars denote the number of patients at the different time-points.

\section{Biochemical measurements}

Serum IGF-I concentration was increased from $11.9 \pm 0.9$ to $39.8 \pm 2.5 \mathrm{nmol} / \mathrm{l}$ after 6 months of treatment $(P<0.001)$, with no change thereafter. During the first 6 months of GH treatment serum PICP concentration doubled from $112.6 \pm 5.4$ to $202.6 \pm 11.5 \mu \mathrm{g} / \mathrm{l}(P<0.001)$, with a steady decline back to baseline $(130 \pm 47 \mu \mathrm{g} / \mathrm{l}$; there was no statistical difference from baseline) during the following 36 months. Serum ICTP concentration behaved similarly. During the first 6 months of treatment it increased from $4.4 \pm 0.3$ to $10.5 \pm 0.6 \mu \mathrm{g} / \mathrm{l} \quad(P<0.001)$ and decreased thereafter to $5.6 \pm 0.7 \mu \mathrm{g} / \mathrm{l}$ at the end of the study (no statistical difference from baseline). As analyzed separately, the changes in serum IGF-I, PICP and ICTP for those 20 patients who completed 42 months of treatment were similar to those presented above for the whole study population (data not shown). Changes in ICTP between 0 and 6 months correlated positively with changes in vertebral BMD between 0 and 30 months $(r=0.357, P<0.05)$. There was a weak inverse correlation between baseline IGF-I levels and changes in femoral neck BMD between 0 and 30 months $(r=-0.112, P<0.05)$.

\section{Comparisons between men and women}

As shown in Fig. 2A-D the BMD increased significantly more in male than in female patients at the lumbar spine $(P<0.05$, ANOVA), the femoral neck $(P<0.001)$ and at the trochanter $(P<0.001)$, but not at the Ward's triangle. Among the 32 patients who completed 36 months of treatment, $40 \%$ of the men had at least osteopenia in the lumbar spine and $60 \%$ at the femoral neck at the start of $\mathrm{GH}$ treatment; the respective percentages for female patients were $25 \%$ in the lumbar spine and 33\% at the femoral neck. After 3 years of $\mathrm{GH}$ treatment the prevalence of osteopenia was halved among male patients and had reduced to 17 and $25 \%$ among female patients in the lumbar spine and in the femoral neck respectively. Of the 20 patients who completed 42 months of treatment, 7 men and 6 women had at least osteopenia at the lumbar spine and 8 men and 5 women at the femoral neck before treatment. After treatment the number of these patients had reduced to three men and three women at the lumbar spine and to two men and three women at the femoral neck.

The responses of serum IGF-I, PICP and ICTP to GH treatment were all more marked in male than 
A. LUMBAR SPINE

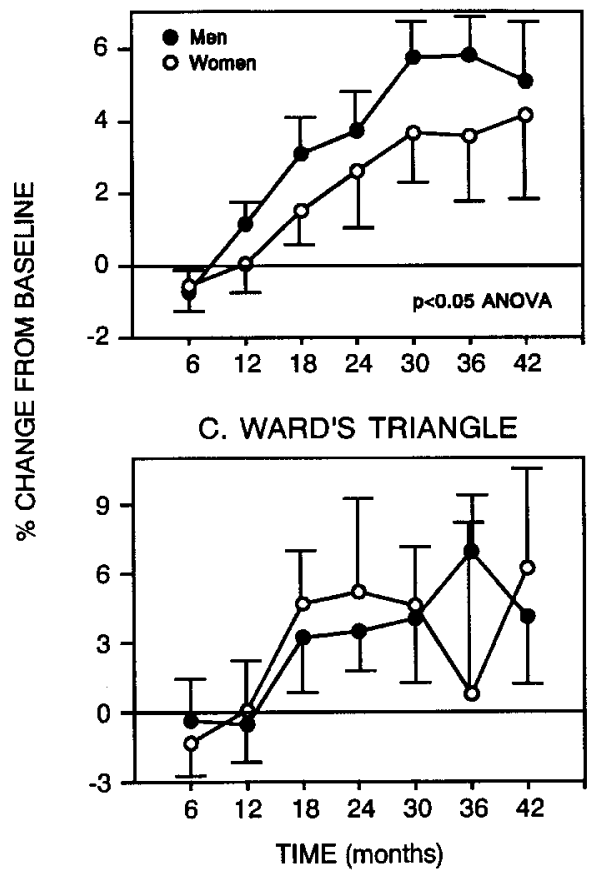

B. FEMORAL NECK

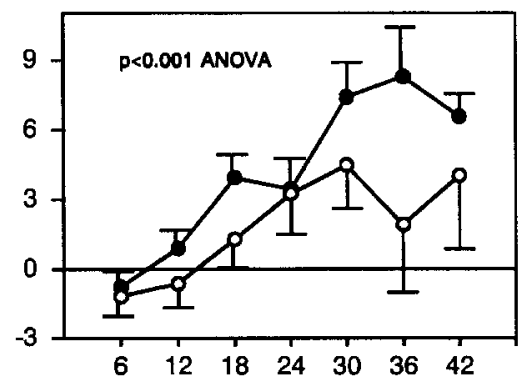

D. TROCHANTER

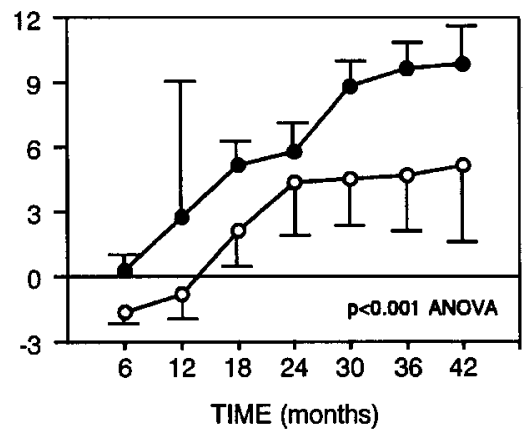

Figure 2 BMD (mean \pm S.E.M.) in response to GH treatment of up to 42 months at the lumbar spine (A), the femoral neck (B), the Ward's triangle $(C)$, and the trochanter area $(D)$ in male and female patients.

female patients $(P<0.001$, ANOVA $)$ (Fig. 3A-C). At baseline only serum PICP was higher in men than in women $(122.2 \pm 7.5$ vs $94.8 \pm 4.9 \mu \mathrm{g} / \mathrm{l}$, $P<0.01)$

\section{Comparisons between patients with low vs high bone mass at baseline}

To study the effect of pretreatment bone mass on BMD in response to GH therapy the patients were divided into low and high bone mass groups on the basis of the presence of osteopenia at the femoral neck at the start of the trial. Consequently, the low bone mass group comprised 31 and the high bone mass group 33 patients at baseline. As shown in Fig. 4A-D, at all the four measurement sites patients with low bone mass demonstrated more marked increases in BMD than patients with high bone mass. After 2 years of treatment the increases in BMD at the four measurement sites ranged from 5.6 to $9.3 \%$ in the low bone mass group $(P<0.01-0.001)$ and from 1.6 to $3.3 \%$ in the high bone mass group (NS). After 3 years the respective changes were 6.3 to $13.0 \%(P<0.01-0.001)$ and 2.3 (NS) to $6.2 \%(P<0.05)$. At the end of the study the BMD had increased by 5.7 to $10.6 \%$ $(P<0.01-0.001)$ in the low bone mass group and by 0.1 to $5.3 \%$ (NS) in the high bone mass group. The statistical significancies for differences between the groups remained the same even when analyzed in terms of absolute changes (data not shown).

\section{Effect of age}

To study the effect of age on the response in BMD to GH treatment the patients were divided into young and old groups on the basis of the median age. As shown in Fig. 5A-D the increases in BMD were more marked in young than in old patients in the lumbar spine $(P<0.05$, ANOVA $)$, the femoral neck $(P<0.05)$ and in the trochanter $(P<0.001)$. Eleven of twelve patients with childhood-onset disease belonged to the younger group of patients. The increases in BMD were more marked in patients with childhood-onset than adultonset GH deficiency in the femoral neck $(P<0.05)$ and in the Ward's triangle $(P<0.05)$.

\section{Multiple regression analysis}

To determine the independent predictors of the response in BMD to $\mathrm{GH}$ treatment stepwise linear regression analysis was performed. As presented in Table 2 the gender and the pretreatment bone mass appeared to be independent predictors at three measurements sites, whereas the age independently determined only the BMD at the lumbar spine. The models calculated explained, however, only from 7 to 

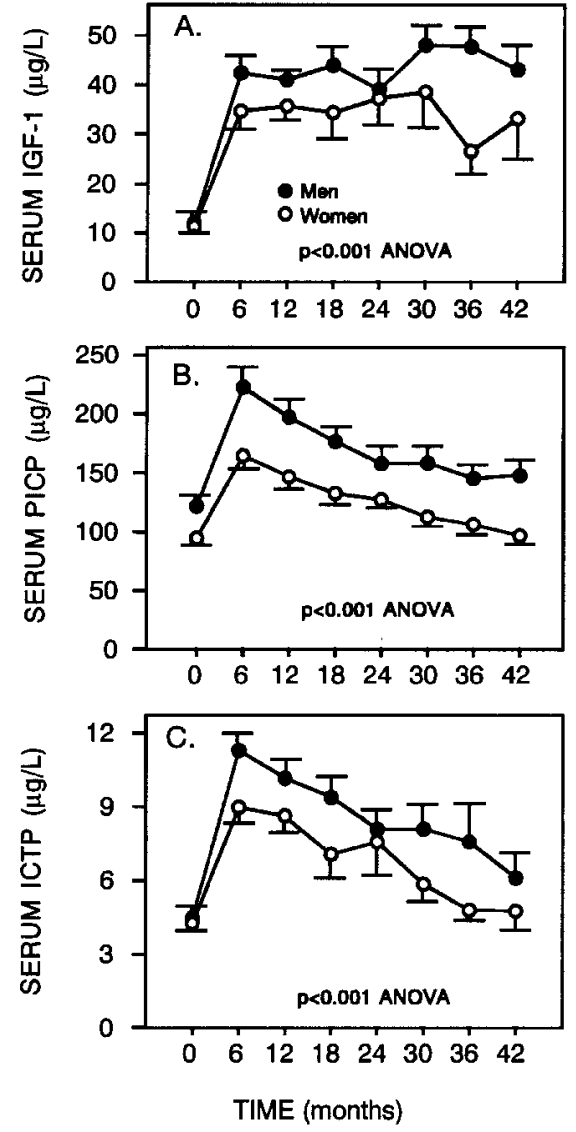

Figure 3 Serum IGF-I (A), PICP (B) and ICTP (C) (mean \pm S.E.M.) in response to $\mathrm{GH}$ treatment of up to 42 months in male and female patients. Normal values: IGF-I between 19 and 25 years of age $15-51 \mathrm{nmol} / \mathrm{l}$, between 26 and 35 years $13.5-44 \mathrm{nmol} / \mathrm{l}$, between 36 and 45 years $11-38 \mathrm{nmol} / \mathrm{l}$, between 46 and 55 years $8-32 \mathrm{nmol} / \mathrm{l}$, over 56 years $5-28 \mathrm{nmol} / /$; PICP in women $50-190 \mu \mathrm{g} / \mathrm{l}$, in men 50-230 $\mu \mathrm{g} / \mathrm{l}$; ICTP $1.5-4.5 \mu \mathrm{g} / \mathrm{l}$.

$31 \%$ of the variation in the response of $\mathrm{BMD}$ to $\mathrm{GH}$ treatment.

\section{Discussion}

At present, the insulin tolerance test is the diagnostic test of choice for GH deficiency (27); most normal subjects show a peak $\mathrm{GH}$ concentration of more than $5 \mu \mathrm{g} / \mathrm{l}$ (28). This test was used only in the minority of the present patients, and in a third of the patients GH deficiency was ascertained with the clonidine test, which is less useful in adults than in children (28). However, we know that among patients with hypothalamic-pituitary disease and one or more additional pituitary deficits, GH deficiency is present in $80-100 \%$ of the patients (29). Ninety-four percent of the present patients needed at least one replacement therapy for other pituitary deficits. This, together with clearly lowered responses in glugacon or clonidine stimulation tests, confirms GH deficiency of our patients. In several subjects GH deficiency was established for the first time just before the study. The median duration of need for other replacement therapies was, however, 8 years in our patients, showing that most likely they also had GH deficiency of a long duration. More than a half of the patients dropped out before the completion of 3 years of treatment. Most subjects interrupted the trial, since they did not subjectively feel benefit from GH injections; only the minority stopped treatment due to adverse effects.

Consistent with previous findings $(12-14,21,22)$ BMD tended to decline during the first 6 months of $\mathrm{GH}$ treatment. Thereafter BMD increased and exceeded the baseline level statistically significantly at the trochanter area at 12 months and at the three other measurement sites at 18 months. In the study of Johansson et al. (18), the $\mathrm{BMD}$ increased by $3.8 \%$ in the lumbar spine, by $4.1 \%$ in the femoral neck, by $5.6 \%$ in the trochanter and by $4.9 \%$ in the Ward's triangle after 2 years of GH treatment. The respective increases of 3.4, 3.4, 5.4 and $4.0 \%$ in the present investigation agree well with these previous data. After 2 years, bone mass in our study increased for up to 30-36 months but plateaued thereafter. A similar plateau in vertebral BMD was not observed in the study of Kann et al. (25) but direct comparisons are made difficult by the fact that instead of percent changes from baseline the results in that study were expressed as percentages of age- and sexrelated normal values (25).

As previously observed (7-19), the markers of bone turnover increased in response to $\mathrm{GH}$. To reflect bone formation we measured serum PICP. Serum osteocalcin and total and bone-specific alkaline phosphatase have been used for the same purpose with similar results $(7-19)$. As a sign of increased bone resorption, serum ICTP rose in our patients in response to $\mathrm{GH}$ treatment. In previous $\mathrm{GH}$ studies, increased bone resorption has been demonstrated using either the same marker $(17,18)$, the urinary excretion of pyridinoline and deoxypyridinoline $(8,19)$, hydroxyproline $(10,14)$ or type I collagen amino-terminal telopeptide (24). Taking all these bone marker findings into account, it seems quite obvious that GH treatment accelerates bone turnover, both bone formation and bone resorption. Recently, the same conclusion was reached in a histomorphometric study in GH-deficient adults (20).

An interesting new finding in our study was the normalization of the serum concentrations of PICP and ICTP after 42 months of GH treatment. The reliability of this finding was supported by the fact that the normalization took place at the same time as the increase in BMD started to level off. A similar normalization of serum ICTP and PICP was not observed in the study of Kann et al. (25) but the maximum increases of the markers were much less in that study than in the present one. On the other 

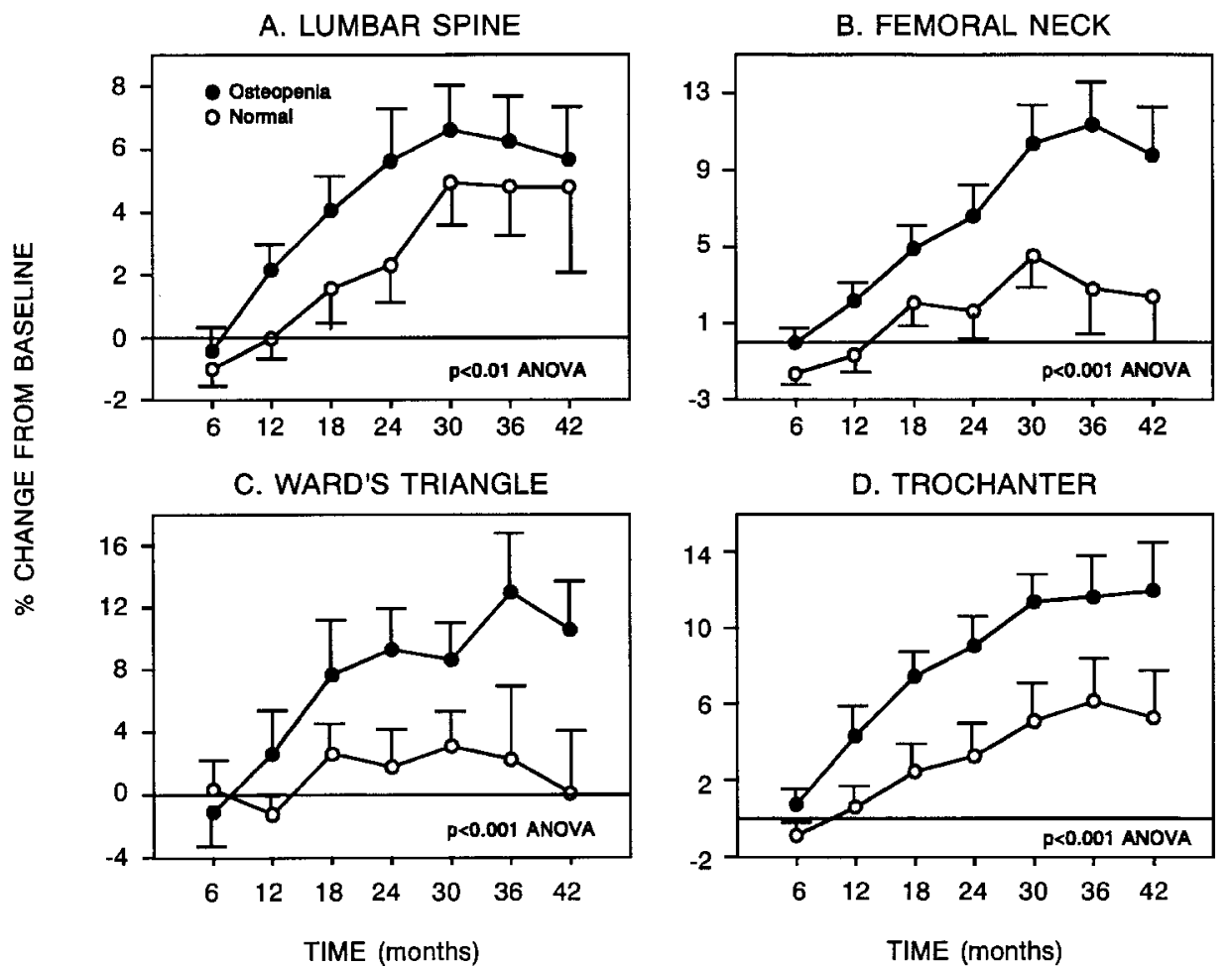

Figure $4 \mathrm{BMD}$ (mean \pm S.E.M.) in response to $\mathrm{GH}$ treatment of up to 42 months at the lumbar spine (A), the femoral neck (B), the Ward's triangle $(C)$ and the trochanter area $(D)$ in patients with at least osteopenia at the femoral neck at baseline and in those with normal bone status.
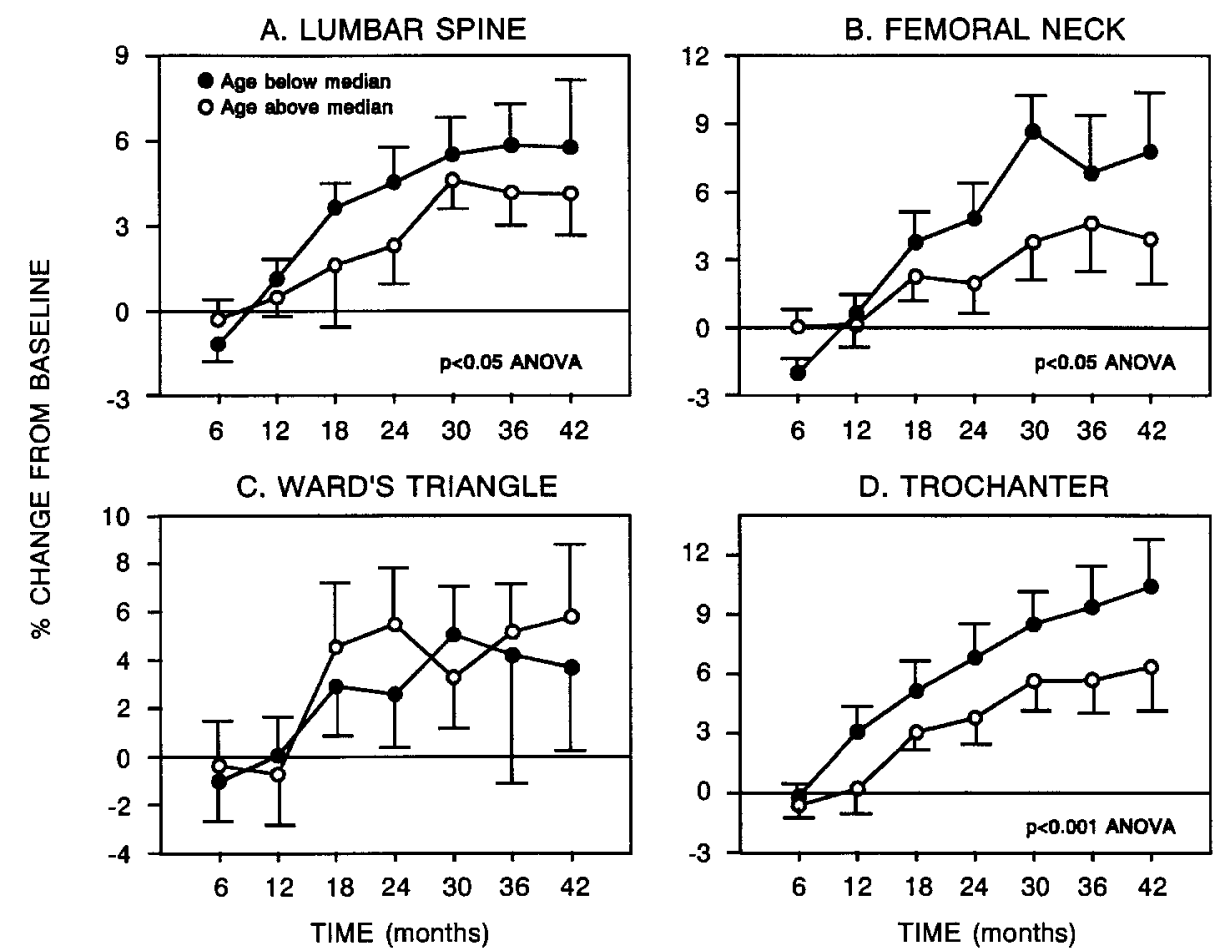

Figure $5 \mathrm{BMD}$ (mean \pm S.E.M.) in response to $\mathrm{GH}$ treatment of up to 42 months at the lumbar spine $(\mathrm{A})$, the femoral neck (B), the Ward's triangle $(C)$ and the trochanter area $(D)$ in patients with age below and above median at baseline. 
Table 2 Dependency of the response (percent change from baseline) in BMD on the duration (months) of GH treatment, gender ( 1 for men, 0 for women), pretreatment bone mass (PBM; 0 for osteopenia, 1 for normal $\mathrm{BMD}$ ), and age (years) on the basis of multiple regression analysis.

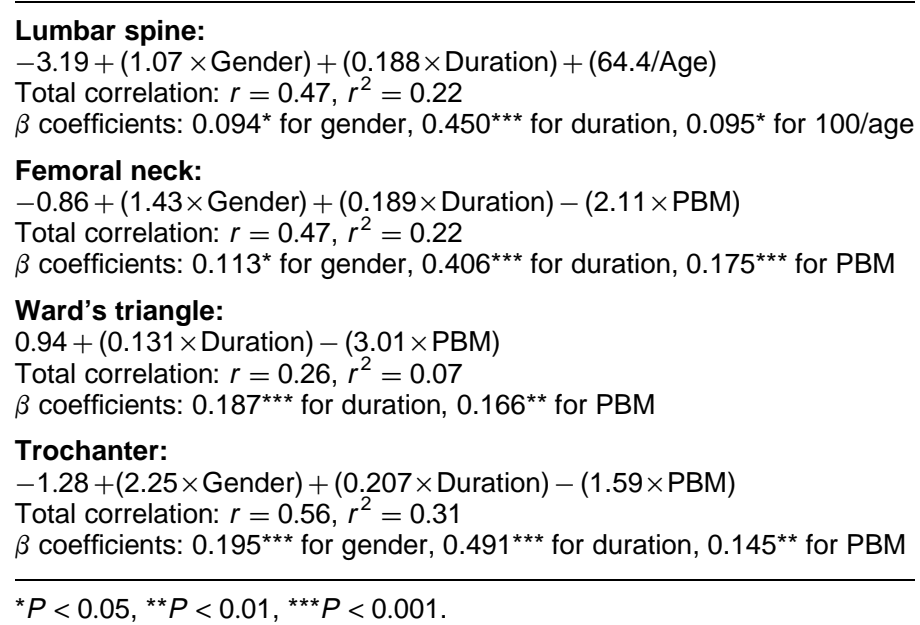

hand, in one study, after 24 months of therapy, serum levels of osteocalcin decreased to pretreatment values while urinary hydroxyproline and serum alkaline phosphatase remained elevated (10). In another study, after an initial rise, serum PICP and osteocalcin were almost back to baseline level after 30 months of $\mathrm{GH}$ treatment (14). Importantly, as in our study the normalization of bone turnover occurred in the face of constantly elevated serum IGF-I levels (14). The mechanism of the dissociation between bone turnover rate and elevated serum IGF-I levels remains totally obscure.

In agreement with the observation of Johansson et al. (18) the increases in serum PICP and ICTP as well as in $\mathrm{BMD}$ in response to $\mathrm{GH}$ treatment were more marked in men than in women. It is also noteworthy that before treatment osteopenia and osteoporosis were more frequent among male than female patients. Thus, estrogens somehow lessen the effects on the skeleton of both GH deficiency and GH replacement therapy. There are several reasons to postulate that bigger doses of $\mathrm{GH}$ are needed in women than in men to accomplish similar bone gain in response to $\mathrm{GH}$ treatment. The mean daily serum concentrations of GH are higher in healthy women than in healthy men (30). In one study the response of serum IGF-I to one s.c. injection of $\mathrm{GH}(0.1 \mathrm{mg} / \mathrm{kg})$ was less in healthy women than men, and in postmenopausal females the response was further lessened by oral estrogen replacement therapy (31). Finally, as shown also in the present study, when $\mathrm{GH}$ doses are determined on a weight basis for GH-deficient adults, the serum IGF-I levels remain lower in women than in men $(18,32)$.

Clinically, it is important that in accordance with two previous studies $(18,33)$ the effect of GH treatment was most prominent in patients with osteopenia or osteoporosis. In the low bone mass group (at least osteopenia), the BMD increased by $6.3 \%$ in the lumbar spine, by $11.4 \%$ in the femoral neck, by $13.0 \%$ in the Ward's triangle and by $11.6 \%$ in the trochanter area after 3 years of GH treatment. Moreover, in more than a half of the patients the criteria of osteopenia disappeared or there was an improvement of the bone state from osteoporosis to osteopenia.

Also the age appeared to determine the response in BMD to GH treatment, with a greater increase in young than in old subjects. Nevertheless, the multiple regression analysis showed that the age seemed to be of less significance than the gender or the pretreatment bone mass, independently determining only the vertebral BMD. In contrast to our finding, no significant correlations were found between the patient's age and the response to treatment in terms of changes in BMD in the study of Johansson et al. (18). A part of the younger patient group in the present study were those with childhood-onset GH deficiency who have a greater potential to respond with an increase in bone mass to $\mathrm{GH}$ treatment than subjects with adult-onset $\mathrm{GH}$ deficiency $(14,22,23)$. In them the beneficial effect of $\mathrm{GH}$ treatment may still be due to an increase in peak bone mass.

The present study indicates that from the skeletal point of view GH-deficient patients exhibiting osteopenia or osteoporosis should be considered candidates for GH supplementation. However, longer-lasting studies are needed to establish whether the positive effects on BMD are persistent and are really associated with a reduction in fracture risk. Furthermore, our findings of the normalization of bone turnover and the plateau in increase of bone mass warrant longer, 
comparative studies, in which after 3-4 years of GH treatment patients are randomized either to continue on treatment or to stop using it.

\section{Acknowledgements}

The study was funded by Pharmacia \& Upjohn, Oy, Vantaa, Finland.

\section{References}

1 Wuster C. Growth hormone and bone metabolism. Acta Endocrinologica 1993128 (Suppl 2) 14-18.

2 Kaufman J-M, Taelman P, Vermeulen A \& Vandeweghe M. Bone mineral status in growth-hormone deficient males with isolated and multiple pituitary deficiencies of childhood onset. Journal of Clinical Endocrinology and Metabolism 199274 118-123.

3 Holmes SJ, Economou G, Whitehouse RW, Adams JE \& Shalet SM. Reduced bone mineral density in patients with adult onset growth hormone deficiency. Journal of Clinical Endocrinology and Metabolism 199478 669-674.

4 Rosen T, Hansson T, Granhed H, Szucs J \& Bengtsson B-Å. Reduced bone mineral content in adult patients with growth hormone deficiency. Acta Endocrinologica 1993129 201-206.

5 Johansson AG, Burman P, Wertermark K \& Ljunghall S. The bone mineral density in acquired growth hormone deficiency correlates with circulating levels of insulin-like growth factor I. Journal of Internal Medicine 1992232 447-452.

6 Rosen T, Wilhelmsen L, Landin-Wilhelmsen K, Lappas G \& Bengtsson B-Å. Increased fracture frequency in adult patients with hypopituitarism and GH deficiency. European Journal of Endocrinology 1997137 240-245.

7 Johansen JS, Pedersen SA, Jorgensen JOL, Riis BJ, Christiansen C, Christiansen JS et al. Effects of growth-hormone $(\mathrm{GH})$ on plasma bone Gla protein in GH-deficient adults. Journal of Clinical Endocrinology and Metabolism 199070 916-919.

8 Schlemmer A, Johansen JS, Pedersen SA, Jorgensen JOL, Hassager C \& Christiansen C. The effect of growth hormone (GH) therapy on urinary pyridinoline cross-links in GH-deficient adults. Clinical Endocrinology 199135 471-476.

9 Bengtsson B-Å, Eden S, Lönn L, Kvist H, Stokland A, Lindstedt G et al. Treatment of adults with growth hormone $(\mathrm{GH})$ deficiency with recombinant human GH. Journal of Clinical Endocrinology and Metabolism 199376 309-317.

10 Balducci R, Toscano V, Pasquino AM, Mangiantini A, Municchi G, Armenise $\mathrm{P}$ et al. Bone turnover and bone mineral density in young adult patients with panhypopituitarism before and after long-term growth hormone therapy. European Journal of Endocrinology $199513242-46$.

11 Whitehead HM, Boreham C, Mcllrath EM, Sheridans B, Kennedy L, Atkinson $\mathrm{AB}$ et al. Growth hormone treatment of adults with growth hormone deficiency: results of a 13-month placebo controlled cross-over study. Clinical Endocrinology 199236 45-52.

12 Amato G, Carella C, Fazio S, LaMontagna G, Cittadini A, Sabatini D et al. Body composition, bone metabolism, and heart structure and function in growth hormone $(\mathrm{GH})$-deficient adults before and after GH replacement therapy at low doses. Journal of Clinical Endocrinology and Metabolism 199377 1671-1676.

13 Thoren M, Soop M, Degerblad M \& Sääf M. Preliminary study of the effects of growth hormone substitution therapy on bone mineral density and serum osteocalcin levels in adults with growth hormone deficiency. Acta Endocrinologica 1993128 (Suppl 2) 1-43.

14 Vandeweghe M, Taelman P \& Kaufman J-M. Short and long-term effects of growth hormone treatment on bone turnover and bone mineral content in adult growth hormone-deficient males. Clinical Endocrinology 199339 409-415.
15 Juul A, Pedersen SA, Sorensen S, Winkler K, Jorgensen JOL, Christiansen JS et al. Growth hormone $(\mathrm{GH})$ treatment increases serum insulin-like growth factor binding protein-3, bone isoenzyme alkaline phosphatase and forearm bone mineral content in young adults with GH deficiency of childhood onset. European Journal of Endocrinology 1994131 41-49.

16 Rosen T, Johansson G, Hallgren P, Caidahl K, Bosaeus I \& Bengtsson B- $\AA$. Beneficial effects of 12 months replacement therapy with recombinant human growth hormone to growth hormone deficient adults. Endocrinology and Metabolism 19941 55-66.

17 Beshyah SA, Kyd P, Thomas E, Fairney A \& Johnston DG. The effects of prolonged growth hormone replacement on bone metabolism and bone mineral density in hypopituitary adults. Clinical Endocrinology 199542 249-254.

18 Johansson G, Rosen T, Bosaeus I, Sjöström L \& Bengtsson B-Å. Two years of growth hormone (GH) treatment increases bone mineral content and density in hypopituitary patients with adultonset GH deficiency. Journal of Clinical Endocrinology and Metabolism 199681 2865-2873.

19 Baum HBA, Biller BMK, Finkelstein IS, Cannistraro KB, Oppenheim DS, Schoenfeld DA et al. Effects of physiologic growth hormone therapy on bone density and body composition in patients with adult-onset growth hormone deficiency. Annals of Internal Medicine 1996125 883-890.

20 Bravenboer N, Holzmann P, De Boer H, Roos JC, Van Der Veen EA \& Lips P. The effect of growth hormone $(\mathrm{GH})$ on histomorphometric indices of bone structure and bone turnover in GH-deficient men. Journal of Clinical Endocrinology and Metabolism $1997821818-1822$.

21 Degerblad M, Elgindy N, Hall K, Sjöberg H-E \& Thoren M. Potent effect of recombinant growth hormone on bone mineral density and body composition in adults with panhypopituitarism. Acta Endocrinologica 1992126 387-393.

22 Holmes SJ, Whitehouse RW, Swindell R, Economou G, Adams JE \& Shalet SM. Effect of growth hormone replacement on bone mass in adults with adult onset growth hormone deficiency. Clinical Endocrinology 199542 627-633.

23 O'Halloran DJ, Tsatsoulis A, Whitehouse RW, Holmes SJ, Adams JE \& Shalet SM. Increased bone density after recombinant human growth hormone $(\mathrm{GH})$ therapy in adults with isolated $\mathrm{GH}$ deficiency. Journal of Clinical Endocrinology and Metabolism 1993 76 1344-1348.

24 Ghiron LJ, Thompson JL, Holloway L, Hintz RL, Butterfield GE, Hoffman AR et al. Effects of recombinant insulin-like growth factor-I and growth hormone on bone turnover in elderly women. Journal of Bone and Mineral Research 199510 1844-1852.

25 Kann P, Piepkorn B, Schehler B, Andreas J, Lotz J, Prellwitz Wet al. Effect of long-term treatment with $\mathrm{GH}$ on bone metabolism, bone mineral density and bone elasticity in GH-deficient adults. Clinical Endocrinology 199845 561-568.

26 Kanis JA, Melton LJ, Christiansen C, Johnston CC \& Khaltaev N. Perspective. The diagnosis of osteoporosis. Journal of Bone and Mineral Research 19949 1137-1141.

27 Hoffman DM, O'Sullivan AJ, Baxter RC \& Ho KK. Diagnosis of growth-hormone deficiency in adults. Lancet 1994343 1046-1068.

28 Invited report of a workshop. Consensus guidelines for the diagnosis and treatment of adults with growth hormone deficiency: summary statement of the growth hormone research society workshop on adult growth hormone deficiency. Journal of Clinical Endocrinology and Metabolism 199883 379381.

29 Toogood AA, Beardwell CG \& Shalet SM. The severity of growth hormone deficiency in adults with pituitary disease is related to the degree of hypopituitarism. Clinical Endocrinology 199441 511-516.

30 Ho KK \& Weissberger JA. Secretory patterns of growth hormone according to sex and age. Hormone Research 199033 (Suppl 4) 7-11. 
31 Lieberman SA, Mitchell AM, Marcus R, Hintz RI \& Hoffman AR. The insulin-like growth factor I generation test: resistance to growth hormone with aging and estrogen replacement therapy. Hormone and Metabolic Research 199426 229-233.

32 Johansson G, Bjarnason R, Bramnert M, Carlsson LMS, Degerblad M, Manhem $\mathrm{P}$ et al. The individual responsiveness to growth hormone $(\mathrm{GH})$ treatment in $\mathrm{GH}$-deficient adults is dependent on the level of GH-binding protein, body mass index, age, and gender. Journal of Clinical Endocrinology and Metabolism 199681 1575-1581.
33 Finkenstedt G, Gasser RW, Hofle G, Watfah C \& Fridrich L. Effects of growth hormone $(\mathrm{GH})$ replacement on bone metabolism and mineral density in adult onset GH deficiency: results of a doubleblind placebo-controlled study with open follow-up. European Journal of Endocrinology 1997136 282-289.

Received 17 September 1998

Accepted 8 February 1999 